\title{
AVALIAÇÃO DE MEIOS FILTRANTES A SEREM EMPREGADOS NA FILTRAÇÃO LENTICULAR DE VINHO TINTO CONSIDERANDO-SE O NÍVEL DE TURBIDEZ DO FILTRADO
}

\author{
E. WENDRINER ${ }^{1}$, M. A. CREMASCO ${ }^{2 *}$ \\ ${ }^{1}$ Onyx Reflexivo \\ ${ }^{2}$ Universidade Estadual de Campinas, Faculdade de Engenharia Química \\ "e-mail: cremasco@feq.unicamp.br
}

\begin{abstract}
RESUMO
$\mathrm{Na}$ produção de vinho existem diversas etapas de filtração, dentre as quais a clarificação, que pode ser realizada por filtro lenticular. Este tipo de filtro caracteriza-se por apresentar-se, fisicamente, na forma de módulos ou de cartuchos, os quais são constituídos de um conjunto de quadros, cada qual contendo dois meios filtrantes. A escolha do meio filtrante depende do que se deseja remover, levando o desempenho da filtração a ser avaliada, também, a partir da qualidade do filtrado. No presente trabalho são apresentados resultados de ensaios de filtração de um tipo vinho tinto de mesa seco, com teor alcoólico de 10,5\%. Em tais ensaios foram utilizados três meios filtrantes, com diferentes tamanho de poros, submetidos à pressão de operação de 15,25 e 30 psi. A qualidade do filtrado foi avaliada considerando-se o nível de turbidez e a contagem de partículas.
\end{abstract}

\section{INTRODUÇÃO}

A produção de vinho ocorre em larga escala em vários países da Europa, como França, Itália, Espanha e Portugal (VARNAM e SUTHERLAND, 1997). No Brasil, a produção de vinho não é tão significativa em comparação a tais países, contudo são produzidos, anualmente, cerca de 250 milhões de litros e com média de consumo de 2 litros per capita/ano. A maior parte desta produção está concentrada no Rio Grande do Sul, que detém $95 \%$ do mercado (FERREIRA et al., 2010).

As características finais de um vinho, como sabor, cor e bouquet são determinadas não só pela composição das uvas, como também pelo processo a que são submetidas. Tais processos envolvem o modo de cultivo e maturação das uvas, manipulação do mosto, condições de fermentação, controle microbiológico, envelhecimento e outros tratamentos (LEA e PIGGOT, 1995). Na elaboração da bebida, a fermentação transforma o açúcar da uva em álcool, impondo modificação significativa no equilíbrio físico-químico do meio. Como consequência, o vinho poderá apresentar, com o passar do tempo, uma série de precipitações ou formações coloidais. Nesse caso, é imprescindível a filtração, uma vez que faz a função de retirar do vinho as partículas sólidas ou coloidais (DIAS, 2007). A eficiência da filtração, portanto, pode ser verificada a partir da medida de parâmetros de qualidade do filtrado como a turbidez e o número total de partículas em suspensão.

No caso da turbidez, verifica-se que as partículas responsáveis por seu aumento, comprometem tanto a qualidade da clarificação quanto a vazão da filtração, pois provocam o entupimento do meio filtrante. 
(RIBÉREAU-GAYON et al., 2006). Um critério utilizado para o envase é o de que o valor da turbidez do vinho deve ser inferior a 1 NTU (Nephelometric Turbidity Unit). Se o vinho apresenta turbidez menor ou igual a 1 NTU, ele está adequado para o engarrafamento, entretanto se o valor for superior a 1, então é recomendado que o vinho passe por um processo extra de filtração antes do envase (BOWYER, 2012).

$\mathrm{Na}$ etapa de redução da turbidez, durante a produção de filtrado de origem microbiológica, é comum utilizar a filtração lenticular, cuja conformação substitui os tradicionais filtros prensa de placa, com a vantagem de reduzir os tempos de troca dos cartuchos, uma vez que apresenta uma construção integral. Este tipo de filtração caracteriza-se por apresentar-se, fisicamente, na forma de módulos ou cartuchos, os quais são constituídos de um conjunto de quadros, cada qual contendo dois meios filtrantes.

No presente trabalho, avaliam-se distintos meios filtrantes, que são submetidos à filtração em escala laboratorial, em diversas condições de pressão de operação. O filtrado obtido é analisado em termos de turbidez e de contagem de partículas.

\section{MATERIAIS E MÉTODOS}

Os ensaios experimentais foram conduzidos na $3 \mathrm{M}$ do Brasil, unidade de Sumaré, São Paulo. Para a realização dos testes experimentais, utilizou-se vinho tinto de mesa seco, da marca Zago, com teor alcoólico de 10,5\%, Safra 2014, Lote A, produzido e engarrafado pela Vinícola Zago Ltda., Videira, Santa Catarina e adquirido diretamente do mercado. As caraterísticas dos meios filtrantes, em termo de diâmetro de poros, estão apresentadas na Tabela 1. Os meios filtrantes apresentam-se na forma de discos de $47 \mathrm{~mm}$ de diâmetro, com matriz celulósica e resina catiônica, fabricada pela
3M do Brasil, Unidade de Mairinque, São Paulo, com a mesma matriz dos cartuchos utilizados no processo de filtração lenticular em escala industrial.

Tabela 1 - Meios filtrantes empregados neste trabalho.

\begin{tabular}{cc}
\hline $\begin{array}{c}\text { Meio filtrante } \\
\text { (nome comercial) }\end{array}$ & $\begin{array}{c}\text { Faixa de tamanho } \\
(\mu \mathrm{m})\end{array}$ \\
\hline $90 \mathrm{~S}$ & 0,05 a 0,30 \\
$60 \mathrm{~S}$ & 0,30 a 0,60 \\
$30 \mathrm{~S}$ & 0,60 a 1,80
\end{tabular}

\subsection{Filtração a pressão constante}

Os meios filtrantes foram submetidos às pressões $(\Delta p)$ de 15,25 e 30 psi e à temperatura de $21^{\circ} \mathrm{C}$, no sistema experimental ilustrado na Figura 1.

Figura 1 - Sistema de filtração

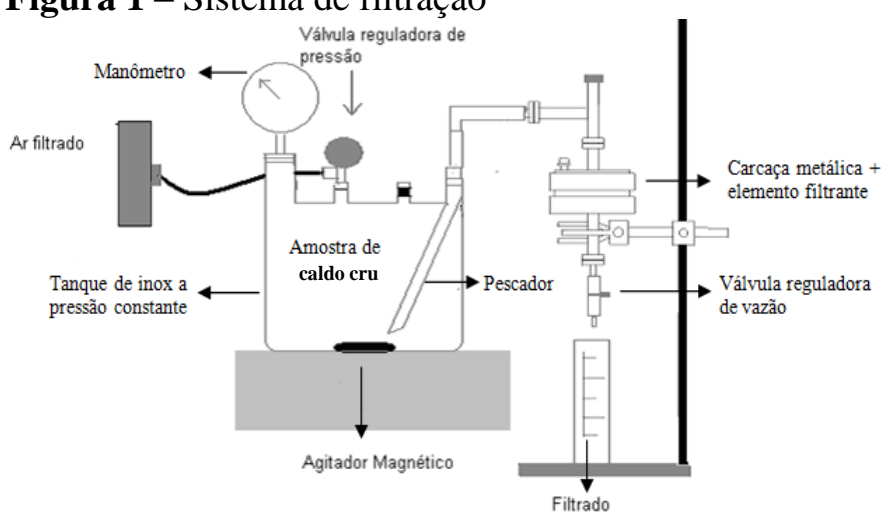

A amostra de vinho cru foi acondicionada em um vaso de pressão de 5 litros, este alimentado por uma mangueira, com ar comprimido, previamente filtrado. Após a pressurização do sistema e abertura da válvula, localizada a jusante da carcaça metálica, o vinho cru atravessou o meio filtrante instalado em seu interior, cujo filtrado foi coletado em uma proveta. No instante em que a primeira gota de filtrado caiu na proveta, o cronômetro foi acionado e o tempo de filtração foi anotado a cada volume de filtrado. A cada valor de pressão, foi obtido um conjunto de valores de tempo de filtração e de volume de filtrado. 


\subsection{Turbidez}

As leituras de turbidez foram feitas em um turbidímetro HACH modelo 2100AN, onde foram introduzidas cubetas de $30 \mathrm{~mL}$, contendo amostras de vinho cru e filtrado, e o equipamento indicava um valor em NTU.

\subsection{Contagem de partículas}

A contagem de partículas foi realizada em um contador de partículas HIAC 9703+. Primeiramente procedeu-se enxágue com água micro filtrada. Em seguida, as amostras foram acondicionadas em frasco específico para realizar a contagem de partículas e imersas no aparelho de ultrassom durante 1 min. A contagem de partículas foi realizada, à temperatura ambiente, segundo indicações do fabricante do equipamento. A cada troca de amostra foi feito um enxágue de três vezes o volume amostrado. $\mathrm{O}$ volume amostrado foi de $20 \mathrm{~mL}$ com vazão através do sensor de 60 $\mathrm{mL} / \mathrm{min}$. As amostras foram contadas três vezes, sendo a primeira contagem descartada.

\section{RESULTADOS}

\subsection{Filtração}

Os ensaios de filtração foram realizados em duplicata para todos os meios filtrantes apresentados na Tabela 1. Com os valores de volume de vinho clarificado, coletados ao longo do tempo, foram construídas as Figuras 2,3 e 4.

A Figura 5 apresenta a produção de filtrado (em $\mathrm{mL} / \mathrm{min}$ ), considerando-se $\mathrm{o}$ volume final de filtrado igual a $600 \mathrm{~mL}$. Observa-se que o incremento da pressão de operação favorece a produção de filtrado, assim como o aumento da micragem do meio filtrante, em particular para pressões superiores a 25 psi quando se compara o desempenho entre $90 \mathrm{~S}$ e $60 \mathrm{~S}$.
Figura 2 - Ensaios de filtração - 90S.

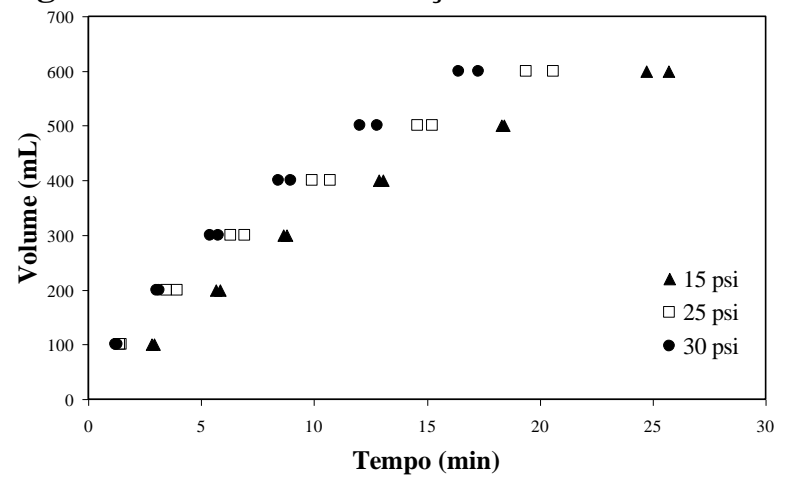

Figura 3 - Ensaios de filtração - 60S.

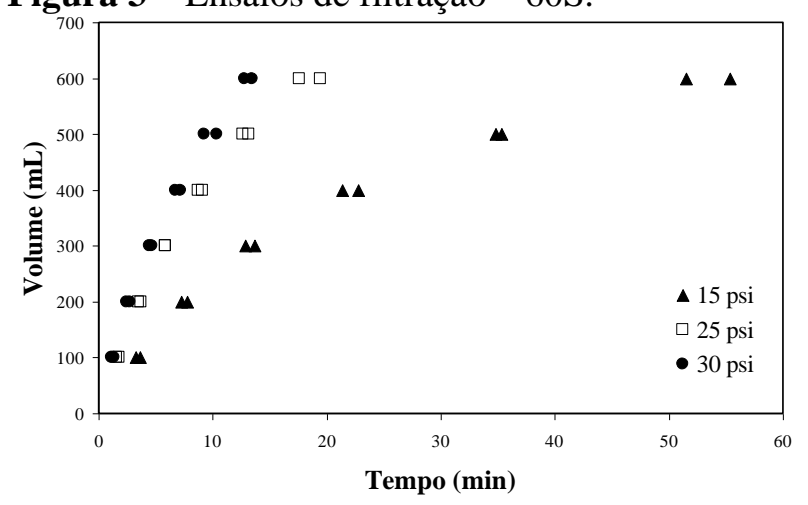

Figura 4 - Ensaios de filtração - 30S.

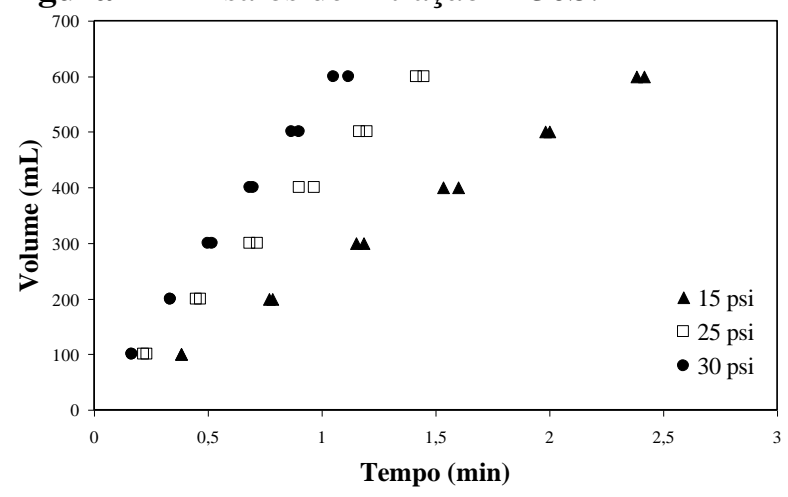

Figura 5 - Produção de filtrado.

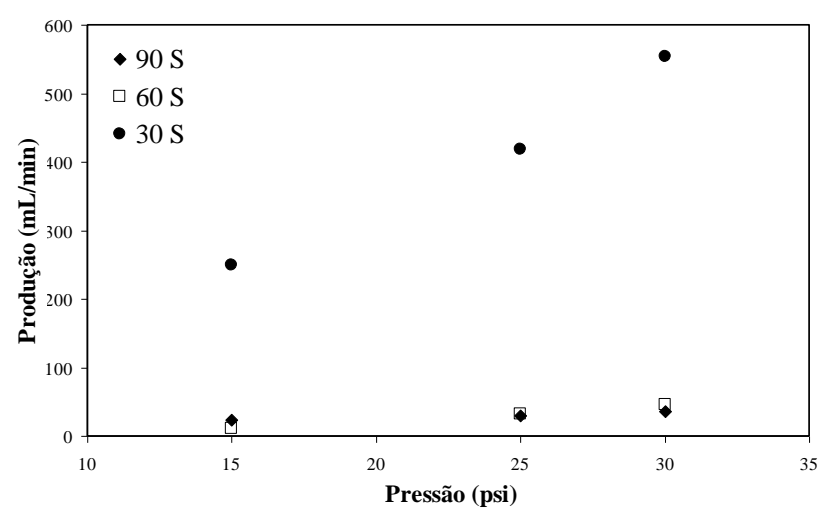




\subsection{Turbidez}

As medidas de turbidez foram realizadas na amostra de vinho cru e aquelas de filtrado, cujos valores encontram-se na Tabela 2. Como os testes de filtração foram realizados em duplicata, os valores representam as médias entre os dois ensaios. A turbidez média para a amostra inicial foi igual a 5,02 NTU.

Tabela 2. Turbidez média para as amostras de vinho após o teste de filtração.

\begin{tabular}{cccc}
\hline $\begin{array}{c}\Delta \mathrm{p} \\
(\mathrm{psi})\end{array}$ & $\begin{array}{c}90 \mathrm{~S} \\
(\mathrm{NTU})\end{array}$ & $\begin{array}{c}60 \mathrm{~S} \\
(\mathrm{NTU})\end{array}$ & $\begin{array}{c}30 \mathrm{~S} \\
(\mathrm{NTU})\end{array}$ \\
\hline 15 & 0,85 & 1,44 & 1,80 \\
25 & 0,94 & 1,56 & 1,92 \\
30 & 1,00 & 1,61 & 2,03
\end{tabular}

Por inspeção da Tabela 2 verifica-se que quanto menor a micragem do meio filtrante, maior a redução da turbidez, uma vez que ocorre maior retenção de partículas na filtração. Além disso, quanto maior a pressão aplicada no processo, maior a turbidez, pois a pressões maiores, facilita-se o trânsito das partículas através do meio filtrante. Considerando-se o parâmetro de turbidez igual a 1 NTU como o valor limite para envase, verifica-se que todas as amostras que foram filtradas pelo elemento $90 \mathrm{~S}$ podem ser consideradas próprias para envase. As demais amostras não poderiam ser imediatamente envasadas, necessitando de etapa extra de filtração.

A partir da Tabela 2 pode-se explicitar a redução de turbidez por meio de

$R_{T}(\%)=\left(1-\frac{N T U_{f}}{N T U_{0}}\right) \times 100$

o que permite a construção da Figura 6, na qual se verifica, para todos o meios filtrantes, redução de turbidez superior a 59\%, sendo que o limite considerado para envase situa-se na faixa superior a $80 \%$, conforme verificado para o meio $90 \mathrm{~S}$.
Figura 6 - Redução de turbidez.

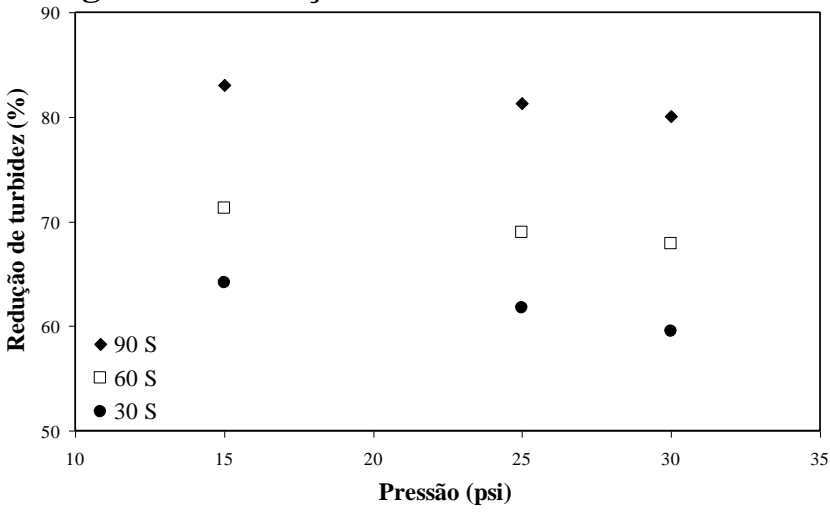

\subsection{Contagem de partículas}

A concentração de partículas encontrada no vinho cru foi igual a 37.709 partículas $/ \mathrm{mL}$. A Tabela 3 apresenta o resultado de contagem de partículas para o vinho filtrado utilizandose os meios filtrantes $90 \mathrm{~S}, 60 \mathrm{~S}$ e $30 \mathrm{~S}$.

Tabela 3 - Concentração de partículas para as amostras de vinho após o teste de filtração.

\begin{tabular}{cccc}
\hline $\begin{array}{c}\Delta \mathrm{p} \\
(\mathrm{psi})\end{array}$ & $\begin{array}{c}90 \mathrm{~S} \\
\left(\mathrm{n}^{\mathrm{o}} . / \mathrm{mL}\right)\end{array}$ & $\begin{array}{c}60 \mathrm{~S} \\
\left(\mathrm{n}^{\mathrm{o}} . / \mathrm{mL}\right)\end{array}$ & $\begin{array}{c}30 \mathrm{~S} \\
\left(\mathrm{n}^{\mathrm{o}} . / \mathrm{mL}\right)\end{array}$ \\
\hline 15 & 10.693 & 11.964 & 12.708 \\
25 & 12.445 & 14.358 & 16.236 \\
30 & 15.210 & 15.710 & 17.337
\end{tabular}

A partir dos resultados apresentados na Tabela 3 verifica-se que a concentração de particulados no filtrado aumenta tanto com o incremento da pressão de operação quanto com o aumento do diâmetro dos poros do meio filtrante, o que se é de esperar, uma vez que tais propriedades auxiliam a passagem de material particulado através do meio filtrante.

De igual maneira à Equação (1), podese avaliar o percentual de remoção de particulados segundo

$$
R_{P}(\%)=\left(1-\frac{c_{f}}{c_{0}}\right) \times 100
$$

De posse da concentração inicial de particulado, $\mathrm{c}_{0}=37.709$ partículas $/ \mathrm{mL}$ e dos valores encontrados na Tabela 3, torna-se possível apresentar a Figura 7. 
Figura 7 - Remoção de particulados.

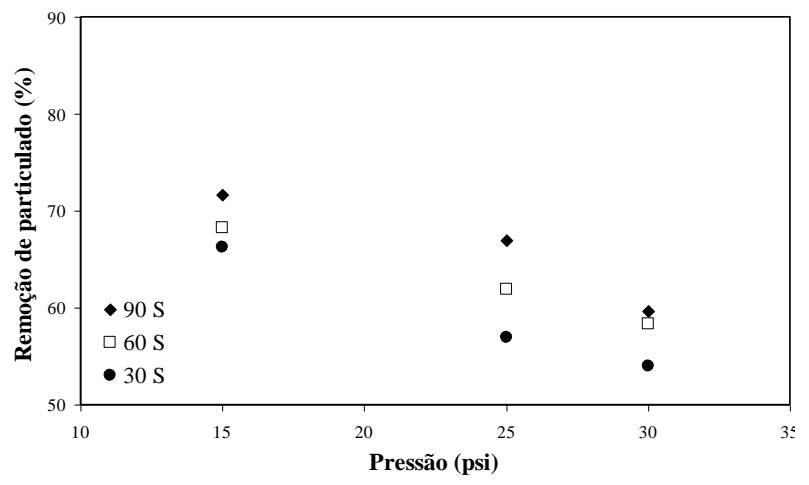

A leitura da Figura 7 conduz a comentários análogos aos proferidos para a Figura 6. Todavia, as diferenças observadas entre os meios filtrantes, no que se refere à remoção de particulados (Figura 7), são menos acentuadas do que a redução de turbidez (Figura 6). Diferentemente da redução de turbidez, na qual o meio filtrante 90S apresenta, em todos os valores de pressão de operação, níveis de redução superiores aos outros meios filtrantes, tal comportamento não se repete quando se analisa a remoção de particulados, conforme pode ser observado pela Figura 8.

Figura 8 - Qualidade do filtrado.

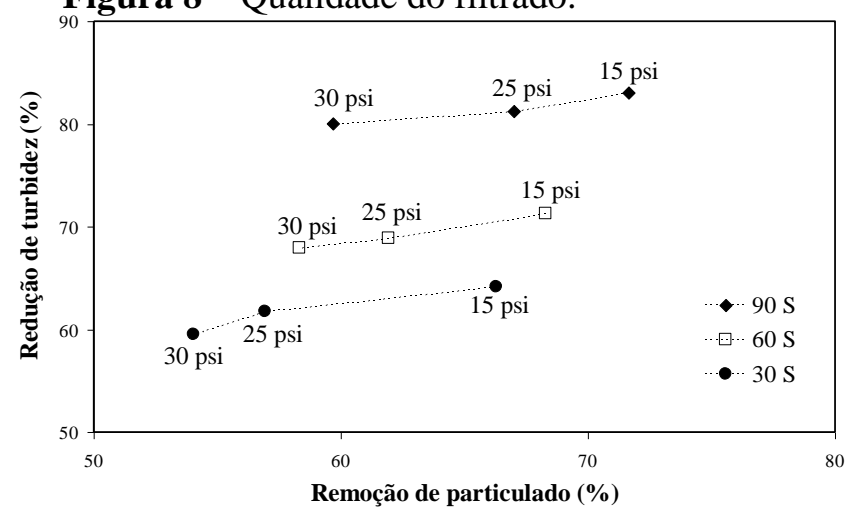

Nota-se, por inspeção da Figura 8, mesmo que se tenha remoção de particulados na ordem de $60 \%$ para todos os meios filtrantes, não implica, necessariamente, na redução de turbidez, dentro do limite de envase. Para o meio 90S, atinge-se $80 \%$ de redução de turbidez, correspondente a 1 NTU, todavia esta redução situa-se na ordem de $60 \%$ para o meio $60 \mathrm{~S}$ e $70 \%$ para o $30 \mathrm{~S}$, os quais correspondem a valores superiores a 1 NTU.

\section{CONCLUSÕES}

Tanto o nível de turbidez quanto a concentração de particulados são influenciados pela natureza da matriz filtrante e da pressão de operação. O aumento do diâmetro dos poros do meio filtrante e da pressão de operação, ainda que aumentem a produção de filtrado, comprometem a sua qualidade, diminuindo os valores de redução de turbidez e de remoção de partículas. Micragem e pressão maiores facilitam a passagem de particulado através do meio filtrante.

Constatou-se que o meio filtrante $90 \mathrm{~S}$, em todas as condições de pressão, conduz ao melhor desempenho quanto à redução da turbidez, apresentando níveis superiores a $80 \%$ e dentro do limite de envase. Verificouse, por outro lado e para o presente trabalho, que a análise apenas da remoção de particulados não é um critério suficiente para a definição de padrão de qualidade.

\section{NOMENCLATURA}

NTU - Nephelometric Turbidity Unit, -; $\Delta \mathrm{p}$ - pressão de operação, psi;

$\mathrm{R}_{\mathrm{P}}$ - percentual de remoção de particulado, -; $\mathrm{R}_{\mathrm{T}}$ - percentual de remoção de turbidez, -;

\section{Subscritos}

0 - vinho cru;

$\mathrm{f}$ - filtrado.

\section{REFERÊNCIAS}

BOWYER, P., EDWARDS, G.; AMELIA E. NTU vs wine filterability index-what does it 
mean for you? Grapegrower \& Winemaker. October 2012. p.76-80.

DIAS, T. Bebidas Livres de Impurezas, Revista Meio Filtrante - Ano V - Edição $\mathrm{n}^{\circ} 25$ - Março/Abril de 2007.

FERREIRA, E. T. D.; ROSINA, C. D.; MOCHIUTTI, F. G. Processo de Produção do Vinho Fino Tinto. In: Encontro de Engenharia de Produção Agroindustrial, 4, 2010, Campo Mourão, Anais... Campo Mourão, PR, FECILCAM, 2012, p. 12.

LEA, A.G.H.; PIGGOTT, J.R. Fermented beverage production. London, Blackie Academic \& Profissional, 1995. p. 121.

RIBÉREAU-GAYON, P.; GLORIES, Y.; MAUJEAN, A.; DUBOURDIEU D. Handbook of Enology: The Chemistry of Wine Stabilization and Treatments. 2 Ed., v. 2, Hoboken, NJ: John Wiley, 2006. p. 339355.

VARNAM, A. H.; SUTHERLAND, J, P. Bebidas: Tecnologia, Química y Microbiologia. Serie 2, Alimentos básicos. Zaragoza: Acribia, 1997. p. 337.

\section{AGRADECIMENTO}

A autora E. Wendriner agradece à $3 \mathrm{M}$ do Brasil, Unidade de Sumaré, SP, pela oportunidade de estágio acadêmico realizado nesta unidade e que possibilitou a feitura de seu Trabalho de Conclusão de curso de graduação na Faculdade de Engenharia Química da Unicamp. 